\title{
ARTIGO DE ATUALIZAÇÃo Disfunção executiva como uma medida de funcionalidade em adultos com TDAH
}

\author{
Executive dysfunction as a measure of \\ functionality in adults with $A D H D$
}

Eloisa Saboya ${ }^{1}$, Dagoberto Saraiva', André Palmini², Pedro Lima², Gabriel Coutinho

\section{RESUMO}

Este artigo aborda a presença e a apresentação clínica da disfunção executiva em pacientes com TDAH. Na medida em que sintomas de disfunção executiva determinam as alterações de funcionamento desses pacientes nas atividades do dia-a-dia, torna-se relevante o estudo das relações entre alterações em testes neuropsicológicos formais de funções executivas e a ocorrência desses sintomas. A constatação relativamente recente do papel da disfunção executiva no TDAH tem impulsionado a pesquisa neuropsicológica, mas os dados até agora disponíveis são ainda pouco consistentes. Uma visão atualizada dessas questões sugere que a semiologia neuropsicológica disponível ainda não alcança plenamente a constelação de sintomas práticos do TDAH no dia-a-dia, mostrando a necessidade de avanços na pesquisa dessas relações.

\section{Palavras-chaves \\ TDAH, atenção, funções \\ executivas, testes \\ neuropsicológicos.}

\section{Key-words}

ADHD, attention, executive function, neuropsychological tests.

\begin{tabular}{c} 
Recebido \\
$31 / 05 / 2007$ \\
Aprovado \\
$01 / 08 / 2007$ \\
\hline
\end{tabular}

1 Instituto de Psiquiatria da Universidade Federal do Rio de Janeiro (UFRJ). 2 Pontifícia Universidade Católica do Rio Grande do Sul (PUC-RS).

Endereço para correspondência: Gabriel Coutinho GEDA - Grupo de Estudos do Déficit de Atenção do Instituto de Psiquiatria da Universidade Federal do Rio de Janeiro Avenida Venceslau Brás, 71, Fundos - 22290-140 - Rio de Janeiro, RJ

E-mail: gabrielccoutinho@yahoo.com.br 


\section{INTRODUÇÃO}

O comprometimento funcional encontrado no transtorno de déficit de atenção/hiperatividade (TDAH) envolve uma diversidade de manifestações, nas quais os vínculos causais ainda não estão bem esclarecidos. A consistência dos achados de comprometimentos no TDAH é reconhecida na literatura científica e na prática clínica; além disso, a presença de comprometimento faz parte dos critérios diagnósticos do DSM-IV (American Psychiatric Association, 1994).

São numerosas as manifestações de comprometimento no TDAH, envolvendo a vida social, familiar, afetiva, conjugal, acadêmica e profissional (Kessler et al., 2006). Subjacentes a esse elenco de impactos funcionais negativos, algumas dificuldades cognitivas pontuais podem também estar presentes, em especial as alterações das funções executivas (Barkley, 1997a). Embora a presença de déficits de funções executivas seja freqüentemente encontrada em portadores de TDAH, tais déficits não são necessários nem suficientes para predizer a presença do transtorno (Willcutt et al., 2005). No entanto, o comprometimento funcional é mais grave em portadores de TDAH com disfunção executiva do que naqueles sem disfunção executiva (Nigg et al., 2005). Essa apresentação aborda as disfunções executivas no TDAH conforme demonstradas por estudos neuropsicológicos, tendo como pano de fundo a importância das funções executivas no desempenho das ações cotidianas e o impacto do comprometimento dessas funções na qualidade de vida.

\section{Consistência}

A validade clínica do comprometimento dos principais âmbitos de vida no TDAH tem sido bastante investigada. Por associar-se a manifestações objetivas, como notas baixas e queixas de comportamento na escola, perda de objetos, acidentes e traumas, esse tipo de comprometimento funcional é freqüentemente reconhecido pelo avaliador, familiares, pessoas do convívio e pelo próprio paciente. No entanto, sua ocorrência deve ser ativamente investigada por meio de entrevista clínica e instrumentos complementares, como escalas de avaliação e testes neuropsicológicos, uma vez que o insight não é a regra (Burges e Alderman, 2004). Por definição, sintomas são manifestações de uma patologia relatadas pelo paciente; assim, o paciente com TDAH nem sempre é rotineiramente sintomático e, ao se queixar dos sintomas, estes, freqüentemente, já podem ser manifestações de uma complicação ou comorbidade (por exemplo, depressão). Na entrevista clínica, deve-se investigar o quanto o paciente tem dificuldade em organizar-se para atingir metas. O clínico poderá interrogá-lo, por exemplo, sobre como administra seu tempo, se é capaz de fazer um planejamento de suas atividades, com que freqüência cumpre seu planejamento, se finaliza tarefas previamente iniciadas e com que grau de autonomia e independência consegue desempenhar suas rotinas.

Os comprometimentos neuropsicológicos no TDAH são amplamente relatados, embora ainda com achados relativamente controversos. Na visão de McGough e Barkley (2004), por exemplo, ainda não há evidências de que testes neuropsicológicos para funções executivas apresentam poder preditivo positivo ou negativo suficiente para justificar seu uso na prática clínica em adultos ou crianças. Os tipos de alterações neuropsicológicas, seus mecanismos, as associações entre os subtipos de TDAH e o próprio comprometimento adaptativo predito por alterações neuropsicológicas não foram ainda caracterizados definitivamente e corroborados na literatura científica. São alterações que exigem investigação mais elaborada, explicando as divergências dos achados cientíícos.

Barkley (1997b) propôs uma teoria unificadora para explicar as disfunções observadas no TDAH. A proposição é pautada em uma alteração central no córtex pré-frontal que compromete a capacidade adaptativa da função executiva. Essa "alteração-chave" seria um déficit na capacidade de inibir respostas, o que explicaria os vários tipos de manifestações e comprometimentos no TDAH. Esse modelo teórico tem encontrado respaldo na literatura científica (Houghton et al., 1999; Bayliss e Roodenrys, 2000). Nesse sentido, alguns autores sugeriram que a divisão por subtipos deveria ser feita de acordo com a presença ou ausência de comprometimento das funções executivas em testes neuropsicológicos, uma vez que os portadores que apresentam tais déficits tendem a ter comprometimentos mais graves e pior desfecho (Nigg et al., 2005). Cumpre ressaltar, no entanto, que outros estudos encontraram resultados divergentes (Shallice et al., 2002).

Déficits relacionados a funções executivas são amplamente descritos nos subtipos de TDAH, embora o comprometimento adaptativo na "vida-real" nem sempre se correlacione com os achados de testes neuropsicológicos. Por outro lado, a identificação de um perfil de alterações em testes neuropsicológicos poderia, em tese, auxiliar na predição, objetivação e quantificação dos impactos negativos do TDAH na condução da vida diária. Dessa forma, o comprometimento neuropsicológico no TDAH - e sua validade ecológica - figura como um aspecto importante a ser pesquisado mais detalhadamente.

\section{Achados neuropsicológicos}

O exame neuropsicológico do portador de TDAH traz alguns benefícios ao processo diagnóstico, apesar de não ser necessário para este, podendo auxiliar o clínico em três questões fundamentais: (1) corroborar o diagnóstico; (2) buscar explicações alternativas para os sintomas apresentados; e (3) identificar condições comórbidas importantes 
de serem tratadas (Gordon e Barkley, 1999). Além disso, ao espelhar dificuldades na condução da "vida real", a avaliação neuropsicológica tem o potencial de antever ou detectar o risco de tais dificuldades, criando as bases para um importante papel de "aconselhamento" acadêmico, ocupacional e social.

As funções executivas podem ser divididas entre quatro subconjuntos: (1) volição; (2) planejamento; (3) ação intencional; e (4) desempenho efetivo (Lezak et al., 2004). A volição é a capacidade de estabelecer objetivos. Para essa formulação intencional, é necessária a motivação e consciência de si e do ambiente. O planejamento é a capacidade de organizar e prever ações para atingir um objetivo. A habilidade de planejar requer capacidade para tomar decisões, desenvolver estratégias, estabelecer prioridades e controlar impulsos. A ação intencional é a efetivação de um objetivo e planejamento, gerando uma ação produtiva. Para isso, é necessário que se inicie, mantenha, modifique ou interrompa um conjunto complexo de ações e atitudes integrada e organizadamente. O desempenho efetivo é a capacidade de automonitorar, autodirigir e auto-regular a intensidade, o ritmo e outros aspectos qualitativos do comportamento e da ação, ou seja, é um controle funcional.

A realização de tarefas diárias e o convívio social adequado requerem a integridade das funções executivas. $O$ desenvolvimento dessas funções durante a infância proporciona gradualmente a adequação e o melhor desempenho da criança para iniciar, persistir e completar tarefas. A identificação de fatores imprevistos e de sua importância, assim como a elaboração de respostas alternativas diante desses problemas, reflete a capacidade adaptativa do indivíduo proporcionada pelas funções executivas (Anderson, 2002).

A síndrome disexecutiva é caracterizada pela incapacidade das funções executivas em processar e elaborar ações adaptadas (Baddeley e Wilson, 1988). Essa disfunção pode se apresentar com uma ou várias dificuldades práticas que impactam o cotidiano, como comprometimento da atenção sustentada, dificuldade em iniciar tarefas, empobrecimento da estimativa de tempo, dificuldade de alternar de uma tarefa para outra ou lidar concomitantemente com distintas tarefas que variam em grau de relevância e prioridade, déficits no controle de impulsos e impaciência, problemas de planejamento, distração, pouco insight, inquietação, agressividade, problemas de seqüência cronológica, problemas de inibição de resposta e labilidade motivacional (Powel e Voeller, 2004; Burges e Alderman, 2004).

Um estudo de revisão sistemática revelou que adultos com TDAH comparados a controles normais apresentam comprometimentos sutis em medidas de funções executivas, baixa velocidade de processamento de informação complexa e déficits atentivos e de aprendizado auditivoverbal (Woods et al., 2002). Uma recente metanálise integrando estudos empíricos que comparam adultos com
TDAH a controles normais em testes neuropsicológicos concluiu que atenção complexa e memória verbal foram as funções que melhor discriminaram os grupos (Schoechlin e Engel, 2005). Outro estudo de metanálise comparando o funcionamento neuropsicológico executivo e não-executivo entre adultos com TDAH e controles normais concluiu que as dificuldades neuropsicológicas dos portadores de TDAH podem estar presentes tanto no domínio executivo quanto no não-executivo (Boonstra et al., 2005).

Recente estudo demonstrou que crianças e adolescentes com TDAH associado a déficits de funções executivas em testes neuropsicológicos estavam sob maiores riscos de ter mau desempenho acadêmico se comparados a portadores sem tais déficits (Biederman et al., 2004). Mais ainda, portadores adultos que apresentam essas disfunções estão mais suscetíveis a ter baixa realização acadêmica, menor status socioeconômico e significativa morbidade funcional do que aqueles portadores sem essas disfunções (Biederman et al., 2006).

Mais recentemente, Biederman et al. (2007) demonstraram que a utilização de um questionário que avaliava comportamentos associados a déficits de funções executivas pode ser útil na identificação de portadores adultos com maiores chances de ter comprometimento funcional. Utilizando o Current Behavior Scale (CBS), um questionário com 99 itens desenvolvido por Barkley (1997a), os autores demonstraram que os portadores que tinham escores mais altos apresentavam comprometimento acadêmico, ocupacional e interpessoal mais graves do que aqueles portadores com escores mais baixos. Esses achados corroboram os achados de estudos que utilizaram testes neuropsicológicos para funções executivas.

A constelação da síndrome disexecutiva no TDAH pode apresentar uma variedade de manifestações. Entre elas, freqüentemente ocorre: (1) procrastinação, ou seja, o indivíduo tende a postergar tarefas, principalmente quando envolvem maior necessidade de atenção ou levam a uma recompensa não a curto, mas a longo prazo; (2) alternância de tarefas, deixando-as incompletas, em função de uma baixa capacidade de persistir em uma tarefa e uma alta necessidade de "variar"; (3) labilidade motivacional, apresentando interesse fugaz com necessidade de buscar novidades (o que se relaciona diretamente com a alternância de tarefas, descrita no item anterior); (4) dificuldade de focalização e sustentação da atenção, revelando maior sensibilidade à distração, com dificuldade para filtrar estímulos internos e externos. Necessita de lembretes para manter uma tarefa habitual, apresentando inconstância e abandono precoce de tarefas; (5) dificuldades de organização e hierarquização, apresentando problemas para estabelecer prioridades e distinguir importâncias; (6) menor velocidade de processamento; (7) manejo deficiente da frustração e da modulação do afeto, apresentando baixa tolerância e limiar para frustração com baixa auto-estima, 
hipersensibilidade a críticas e irritabilidade; (8) deficiência de memória de trabalho com dificuldade de manipular informações verbais e não-verbais em curto espaço de tempo, e seguimento de seqüências; (9) deficiência de memória prospectiva, gerando esquecimentos de responsabilidades e objetivos estipulados (Lopes et al., 2005).

Como mencionado acima, um corpo de dados começa a ser acumulado sobre as correlações entre dificuldades na vida diária e o desempenho em testes neuropsicológicos de função executiva. Por exemplo, Stavro et al. (2007) estudaram 105 adultos com TDAH e 90 controles em termos de comprometimento adaptativo (definido como prejuízo nas esferas social, ocupacional e educacional) e sua relação com a performance em testes neuropsicológicos de funções executivas. Os achados sugeriram que os sintomas de desatenção eram mais duradouros e estariam mais associados ao comprometimento de funções executivas e comprometimento adaptativo.

Lawrence et al. (2004) compararam crianças com e sem TDAH em testes neuropsicológicos e atividades específicas na "vida-real". O grupo com TDAH apresentou comprometimento de funções executivas e velocidade de processamento tanto nos testes neuropsicológicos quanto nas atividades habituais estudadas. Além disso, o desempenho nos testes neuropsicológicos das crianças com TDAH apresentou relação com a eficiência nas atividades habituais estudadas.

\section{CONCLUSÕES}

Conforme o panorama aqui apresentado, pode-se depreender a importância de uma abordagem clínica e neuropsicológica do TDAH que enfatize as disfunções executivas, tendo em vista o seu impacto no desempenho funcional do paciente e, por extensão, em sua qualidade de vida. A significância clínica do comprometimento funcional do TDAH é determinada pelo impacto dos sintomas sobre o desenvolvimento cognitivo e social da criança, bem como por sua influência na autonomia funcional do indivíduo adulto. Portanto, o entendimento do TDAH como uma síndrome disexecutiva permite integrar os achados neuropsicológicos e clínicos de modo a conduzir a uma abordagem mais compatível com a realidade vivida por esses pacientes no seu cotidiano.

Potenciais conflitos de interesse: O GEDA - UFRJ recebe suporte de pesquisa do Laboratório Janssen-Cilag.

\section{REFERÊNCIAS}

American Psychiatric Association. Diagnostic and Statistical Manual of Mental Disorders. $4^{\text {th }}$ ed. Washington, DC: Author; 1994.
Anderson V. Executive function in children. Child Neuropsychology, 8:69-70, 2002

Baddeley AD, Wilson B. Frontal amnesia and the dyexecutive syndrome. Brain \& Cogn, 7 : 212-30, 1988.

Barkley RA. ADHD in adults: comorbidity and adaptive impairments. NIMH Grant number1R01MH054509-01A2, 1997a.

Barkley RA. Behavioral inhibition, sustained attention, and executive functions: Constructing a unifying theory of ADHD. Psychological Bulletin, 121:65-94, 1997b.

Bayliss DM, Roodenrys S. Executive processing and attention deficit hyperactivity disorder: An application of the supervisory attentional system. Dev Neuropsychol, 17:161-80, 2000.

Biederman J, Monuteaux M, Seidman L, Doyle AE, Mick E, Wilens T et al. Impact of executive function deficits and ADHD on academic outcomes in children. J Consult Clin Psychol, 72:757-66, 2004

Biederman J, Petty C, Fried R, Fontanella J, Doyle A, Seidman L et al. Impact of psychometrically defined deficits of executive functioning in adults with Attention Deficit Hyperactivity Disorder. Am J Psychiatry, 163:1730-8, 2006.

Biederman J, Petty C, Fried R, Fontanella J, Doyle A, Seidman L et al. Can self-reported behavioral scales assess executive function deficits? A controlled study of adults with ADHD. J Nerv Ment Dis, 195:240-6, 2007

Boonstra AM, Oosterlaan J, Sergeant JA, Buitelaar JK. Executive functioning in adult ADHD: a meta-analytic review. Psychol Med, 35:1097-108, 2005.

Burges PW, Alderman N. Executive dysfunction. In: Goldstein L, McNeil J, org. Clinical Neuropsychology: A practical guide to assessment and management for clinicians. England: John Wiley \& Sons Ltd, pp. 185-270, 2004.

Gordon M, Barkley RA. Tests and Observational Measures. In Barkley RA, editor. Attention Deficit Hyperactivity Disorder - A Handbook for Diagnosis and Treatment, 2nd ed. New York; The Guilford Press, pp. 294-311, 1999.

Houghton S, Douglas G, West J, Whiting K, Wall M, Langsford S et al. Differential patterns of executive function in children with attention deficit hyperactivity disorder according to gender and subtype. J Child Neurol, 14:801-5, 1999.

Kessler RC, Adler L, Barkley R, Biederman J, Conners CK, Demler 0 et al. The prevalence and correlates of adult ADHD in the United States: results from the National Comorbidity Survey Replication. Am J Psychiatry, 163(4):716-23, 2006.

Lawrence V, Houghton S, Douglas G, Durkin K, Whiting K, Tannock R. Executive function and ADHD: a comparison of children's performance during neuropsychological testing and real-world activities. J Atten Disord, 7:137-149, 2004.

Lezak MD, Howieson DB, Loring DW. Neuropsychological assessment. $4^{\text {th }}$ ed. New York: Oxford University Press; 2004.

Lopes RMF, Nascimento RFL, Bandeira DR. Avaliação do transtorno de déficit de atenção/hiperatividade em adultos (TDAH): uma revisão de literatura. Aval Psicol, 4:65-74, 2005.

McGough JJ, Barkley RA. Diagnostic controversies in adult attention deficit hyperactivity disorder. Am J Psychiatry, 161:1948-56, 2004.

Nigg JT, Stavro G, Ettenhoffer M, Hambrick DZ, Miller T, Henderson JM. Executive Functions and ADHD in Adults: Evidence for Selective Effects on ADHD Symptom Domains. J Abnormal Psychology, 114(3):706-17, 2005.

Powell KB, Voeller KKS. Prefrontal executive function syndromes in children. J Child Neurol, 19:785-97, 2004

Schoechlin C, Engel RR. Neuropsychological performance in adult attention-deficit hyperactivity disorder: meta-analysis of empirical data. Arch Clin Neuropsychol, 20:727-44, 2005.

Shallice T, Marzocchi GM, Coser S, Del Savio M, Meuter RF, Rumiati RI. Executive function profile of children with attention déficit hyperactivity disorder. Dev Neuropsychol, 21: 43-71, 2002

Stavro GM, Ettenhofer ML, Nigg JT. Executive functions and adaptive functioning in young adult attention-deficit/hyperactivity disorder. J Inter Neuropsychol Soc, 13:324-34, 2007.

Willcutt EG, Doyle AE, Nigg JT, Faraone SV, Pennington BF. Validity of the executive theory of ADHD: a meta-analytic review. Biol Psychiatry, 57:1336-46, 2005.

Woods P, Lovejoy DW, Ball JD. Neuropsychological characteristics of adults with ADHD: A comprehensive review of initial studies. Clin Neuropsychol, 16:12-34, 2002. 